\title{
Improving chemotherapeutic drug penetration in tumors by vascular targeting and barrier alteration
}

\author{
Flavio Curnis, Angelina Sacchi, and Angelo Corti \\ Department of Biological and Technological Research, San Raffaele Hospital Scientific Institute, Milan, Italy
}

\begin{abstract}
Drug delivery and penetration into neoplastic cells distant from tumor vessels are critical for the effectiveness of solid-tumor chemotherapy. We have found that targeted delivery to tumor vessels of picogram doses of TNF- $\alpha$ (TNF), a cytokine able to alter endothelial barrier function and tumor interstitial pressure, enhances the penetration of doxorubicin in tumors in murine models. Vascular targeting was achieved by coupling TNF with CNGRC, a peptide that targets the tumor neovasculature. This treatment enhanced eight- to tenfold the therapeutic efficacy of doxorubicin, with no evidence of increased toxicity. Similarly, vascular targeting enhanced the efficacy of melphalan, a different chemotherapeutic drug. Synergy with chemotherapy was observed with 3-5 ng/kg of targeted TNF (intraperitoneally), about $10^{6}$-fold lower than the $\mathrm{LD}_{50}$ and $10^{5}$-fold lower than the dose required for nontargeted TNF. In addition, we have also found that targeted delivery of low doses of TNF to tumor vessels does not induce the release of soluble TNF receptors into the circulation. The delivery of minute amounts of TNF to tumor vessels represents a new approach for avoiding negative feedback mechanisms and preserving its ability to alter drug-penetration barriers. Vascular targeting could be a novel strategy for increasing the therapeutic index of chemotherapeutic drugs.
\end{abstract}

J. Clin. Invest. 110:475-482 (2002). doi:10.1172/JCI200215223.

\section{Introduction}

To reach cancer cells in solid tumors, chemotherapeutic drugs must enter the tumor blood vessels, cross the vessel wall, and finally migrate through the interstitium. Heterogeneous tumor perfusion, vascular permeability and cell density, and increased interstitial pressure could represent critical barriers that may limit the penetration of drugs into neoplastic cells distant from tumor vessels and, consequently, the effectiveness of chemotherapy (1). Strategies aimed at improving drug penetration in tumors are, therefore, of great experimental and clinical interest.

A growing body of evidence suggests that TNF- $\alpha$ (TNF), an inflammatory cytokine endowed with potent antitumor activity, could be exploited for this purpose. For example, the addition of TNF to regional isolated limb perfusion with melphalan or doxorubicin has produced higher response rates in patients with extremity soft-tissue sarcomas or melanomas than those obtained with chemotherapeutic drugs alone (2-6). TNF-induced

Received for publication February 7, 2002, and accepted in revised form June 18, 2002.

Address correspondence to: Angelo Corti,

Immunobiotechnology Unit, Department of Biological and

Technological Research, San Raffaele Hospital Scientific Institute, via Olgettina 58, 20132 Milan, Italy. Phone: 39-022643-4802; Fax: 39-02-2643-4786; E-mail: corti.angelo@hsr.it. Conflict of interest: No conflict of interest has been declared. Nonstandard abbreviations used: TNF- $\alpha$ (TNF); Thy 1.1 cDNA-transfected RMA cells (RMA-T); murine TNF (mTNF); human TNF (hTNF); soluble TNF receptors (sTNF-Rs); soluble p55-TNF receptor (sTNF-R1); soluble p75-TNF receptor (sTNF-R2). alteration of endothelial barrier function, reduction of tumor interstitial pressure, increased chemotherapeutic drug penetration, and tumor vessel damage are believed to be important mechanisms of the synergy between TNF and chemotherapy (3, 4, 7-10). Unfortunately, systemic administration of TNF is accompanied by prohibitive toxicity, the maximum tolerated dose $(8-10 \mu \mathrm{g} / \mathrm{kg})$ being $10-50$ times lower than the estimated effective dose $(11,12)$. For this reason, systemic administration of TNF has been abandoned and the clinical use of this cytokine is limited to locoregional treatments. Nevertheless, some features of the TNF activity, in particular the selectivity for tumor-associated vessels and the synergy with chemotherapeutic drugs, have continued to nourish hopes regarding the possibility of wider therapeutic applications (13).

The vascular effects of TNF provide the rationale for developing a "vascular targeting" strategy aimed at increasing the local efficacy and at enabling systemic administration of therapeutic doses. We have shown recently that targeted delivery of TNF to tumor vessels can be achieved by coupling this protein with the CNGRC peptide, an aminopeptidase N (CD13) ligand that targets the tumor neovasculature (14). In the present work, we have investigated whether vascular targeting with low doses of this conjugate, called NGR-TNF, could enhance the penetration of chemotherapeutic drugs in tumors and improve their efficacy. We show that systemic administration to mice of picogram doses of NGR-mTNF (3-5 ng/ $\mathrm{kg}$ ), six orders of magnitude lower than the $\mathrm{LD}_{50}$, is sufficient to enhance the antitumor activity of melphalan and 
doxorubicin, with no evidence of increased toxicity. In addition, we provide evidence that vascular targeting with NGR-TNF can reduce drug-penetration barriers and increase the amount of doxorubicin that reaches cancer cells. Finally, we show that the delivery of minute amounts of NGR-TNF to tumor vessels overcomes another major problem associated with systemic administration of relatively high doses of TNF, i.e., the induction of soluble TNF inhibitors.

\section{Methods}

Tumor cell lines and reagents. Mouse $\mathrm{B} 16 \mathrm{~F} 1$ melanoma and Thy 1.1 cDNA-transfected RMA cells (RMA-T) lymphoma were cultured as described previously $(14,15)$.

The mAb 6G1 (rat anti-p75 murine TNF [mTNF] receptor antagonist) was produced and characterized as described previously $(16,17)$. The $\mathrm{mAb} V 1 \mathrm{q}$ (rat antimTNF) was kindly supplied by D. Mannel (University of Regensburg, Regensburg, Germany). Melphalan (Alkeran) was obtained from Glaxo Wellcome Operations (Dartford, United Kingdom). Doxorubicin (Adriblastina) was purchased from Pharmacia \& Upjohn SpA (Milan, Italy).

Preparation of human and murine TNF and NGR-TNF. Human and murine TNF and NGR-TNF (consisting of TNF fused with the C-terminus of CNGRCG) were prepared by recombinant DNA technology and purified from Escherichia coli cell extracts, as described (14). All solutions used in the chromatographic steps were prepared with sterile and endotoxin-free water (SALF Laboratorio Farmacologico SpA, Bergamo, Italy). Protein concentration was measured using the BCA Protein Assay Reagent (Pierce Chemical Co., Rockford, Illinois, USA). The in vitro cytolytic activity of human TNF (hTNF), estimated from a standard cytolytic assay with L-M mouse fibroblasts (18), was $5.4 \times 10^{7} \mathrm{U} / \mathrm{mg}$, whereas that of purified NGR-hTNF was $1.4 \times 10^{8} \mathrm{U} / \mathrm{mg}$. The cytolytic activity of mTNF was $7.6 \times 10^{7} \mathrm{U} / \mathrm{mg}$, whereas that of NGR-mTNF was $9.1 \times 10^{7} \mathrm{U} / \mathrm{mg}$. The hydrodynamic volumes of NGR-mTNF, NGR-hTNF, and mTNF were similar to those of hTNF, a homotrimeric protein (19), by gel filtration chromatography on a Superdex 75 HR column (Amersham Biosciences Europe GmbH, Freiburg, Germany). Electrospray mass spectrometry of each product determined the following molecular masses: NGR-hTNF, 17,937.6 $\pm 1.9 \mathrm{Da}$ (expected for CNGRCG-hTNF ${ }_{1-157}$ monomers, 17,939.4 $\mathrm{Da}$ ); hTNF, 17,349 \pm 1.3 (expected for $\mathrm{hTNF}_{1-157}$, 17,350.7); NGR-mTNF, 17,841.16 \pm 2.5 (expected for CNGRCG-mTNF $\left._{1-156}, 17,844.2\right) ;$ mTNF, 17,384.9 \pm 2 (expected for Met-mTNF ${ }_{1-156}, 17,386.7$ ). The endotoxin content of each product, measured using the quantitative chromogenic limulus amoebocyte lysate (LAL) test (BioWhittaker Inc., Walkersville, Maryland, USA), was: NGR-hTNF, $0.079 \mathrm{U} / \mu \mathrm{g}$; hTNF, $0.117 \mathrm{U} / \mu \mathrm{g}$; NGR-mTNF, $0.082 \mathrm{U} / \mu \mathrm{g} ; \mathrm{mTNF}, 1.61 \mathrm{U} / \mu \mathrm{g}$.

In vivo studies. Studies on animal models were approved by the Ethical Committee of the San Raffaele $\mathrm{H}$ Scientific Institute and performed according to the prescribed guidelines. C57BL/6 mice (Charles River Laboratories, Calco, Italy) weighing 16-18 g were challenged with subcutaneous injection in the left flank of $5 \times 10^{4}$ RMA-T or B16F1 living cells; 4-12 days later, the mice were treated with TNF or NGR-TNF solutions $(100 \mu \mathrm{l})$ followed 2 hours later by administration of melphalan or doxorubicin solution $(100 \mu \mathrm{l})$. Unless specified, all drugs were administered intraperitoneally. All drugs were diluted with $0.9 \%$ sodium chloride, containing $100 \mu \mathrm{g} / \mathrm{ml}$ endotoxin-free HSA (FarmaBiagini SpA, Lucca, Italy), except for doxorubicin, which was diluted with $0.9 \%$ sodium chloride alone. Tumor growth was monitored daily by measuring the tumors with calipers as previously described (20). Animals were sacrificed before the tumors reached 1.0-1.5 $\mathrm{cm}$ in diameter. Tumor sizes are shown as mean $\pm \mathrm{SE}$ (five animals per group). Statistical analysis was performed by two-tailed $t$ test. Differences between groups were considered significant when $P$ was less than 0.05 .

Soluble TNF receptor assays. Soluble p55TNF receptor (sTNF-R1) and soluble p75TNF receptor (sTNF-R2) in animal sera were measured using the Quantikine $M$ kit (R\&D Systems Inc., Minneapolis, Minnesota, USA).

Detection of doxorubicin in tumors. C57BL/ 6 mice bearing B16F1 or RMA-T tumors (diameter $0.5-1 \mathrm{~cm}$ ) were treated with NGR-TNF $(0.1 \mathrm{ng}$ diluted in $0.9 \%$ sodium chloride containing $100 \mu \mathrm{g} / \mathrm{ml} \mathrm{HSA}$, intraperitoneally), or with diluent alone, followed 2 hours later by doxorubicin $(320 \mu \mathrm{g}$ intraperitoneally). After 2 hours, the animals were sacrificed and the tumors were excised. Each tumor was weighed, disaggregated, resuspended in cold PBS, and filtered through $70-\mu \mathrm{m}$ filters. The cells were resuspended in cold PBS (50 ml), centrifuged $\left(460 \mathrm{~g}, 10\right.$ minutes, $\left.4^{\circ} \mathrm{C}\right)$, resuspended in cold PBS $(2.5$ $\mathrm{ml} / \mathrm{g}$ of tumor tissue), and mixed with freshly prepared PBS containing $8 \%$ formaldehyde $(2.5 \mathrm{ml} / \mathrm{g}$ of tissue). The cells were stored in the dark at $4{ }^{\circ} \mathrm{C}$ overnight, and then analyzed by FACS. The FACScan (BD Biosciences, Erembodegen, Belgium) was calibrated with cells recovered from untreated tumors. Each sample was then analyzed using the FL-3 filter and CellQuest (BD Biosciences) software.

\section{Results}

Dose-response curves of NGR-mTNF and mTNF in murine lymphoma and melanoma models. The antitumor activity of NGR-mTNF and mTNF was first characterized in the absence of chemotherapeutic drugs. To compare the dose-response curves of NGR-mTNF and mTNF, we performed several experiments based on single or repeated intraperitoneal administration of various doses of NGR-mTNF and mTNF (from 0.01 to 10000 ng) to RMA-T lymphoma- or B16F1 melanoma-bearing mice. Murine TNF delayed tumor growth when administered at high doses (10,000 ng) (Figure 1a); no effects were induced by doses lower than $100 \mathrm{ng}$, either with single (Figure 1a) or with repeated administrations (Figure 1b). NGR-mTNF was markedly more potent. In this case we observed antitumor effects even 

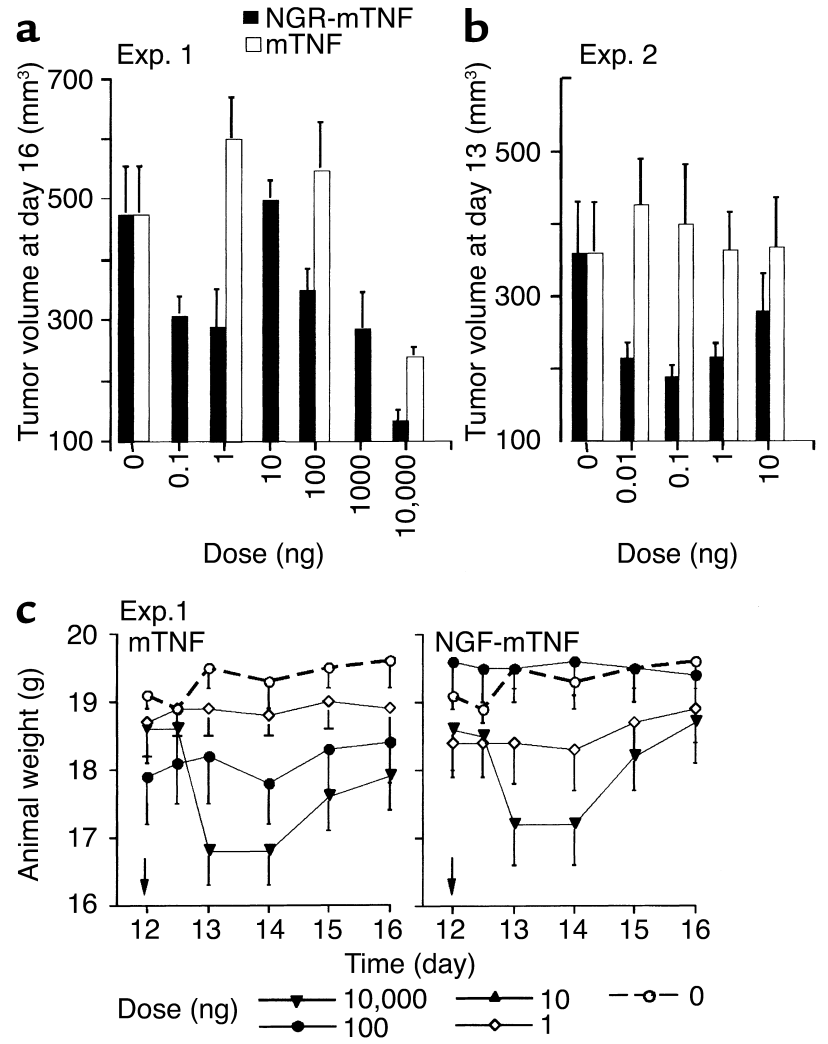

with doses as low as $0.01 \mathrm{ng}$ (Figure 1, a and b). However, the dose-response curve was more complex. For instance, the effect of $10 \mathrm{ng}$ was surprisingly lower than that of $0.01-0.1 \mathrm{ng}$ and $1,000-10,000 \mathrm{ng}$. A bell-shaped dose-response curve was observed in several other experiments conducted in the RMA-T model as well as in the B16F1 melanoma model (not shown). These results suggest that the efficacy of low doses of NGRmTNF is markedly higher than that of mTNF, and that doses of NGR-mTNF greater than 1-10 ng activate negative feedback mechanisms that inhibit its potential antitumor activity.

Nanogram, but not picogram, doses of NGR-TNF induce soluble TNF receptor shedding. The protective mechanisms responsible for the bell-shaped dose-response curve of NGR-mTNF were then investigated. Since exogenously administered TNF can induce shedding of soluble TNF receptors (sTNF-Rs) shedding in vivo (21), we

\section{Figure 2}

Circulating levels of sTNF-R2 and their role in regulating the activity of NGR-mTNF and NGR-hTNF. (a) Serum levels of sTNF-R1 and sTNF-R2 in B16F1 tumor-bearing mice 1 hour after treatment with various doses of NGR-mTNF or mTNF. Animals (three mice per group) were treated at day 6. (b) Effect of the anti-sTNF-R2 mAb 6G1 on the antitumor activity of NGR-mTNF. The mAb 6G1 (100 $\mu \mathrm{g}$ ) was administered to animals bearing B16F1 tumors at day 5 and 8. Each animal was treated 1 hour later with NGR-mTNF at the indicated doses, and 2 hours later with melphalan $(90 \mu \mathrm{g}$, five mice per group). (c) Effect of NGR-hTNF and hTNF on the growth of RMA-T tumors. Mice were treated with various doses of each cytokine at day 11. NS, not significant ( $t$ test).

\section{Figure 1}

Effect of mTNF and NGR-mTNF on tumor growth and body weight of animals bearing RMA-T lymphomas. Animals bearing RMA-T tumors (five mice per group) were treated intraperitoneally with NGR-mTNF or mTNF at day 12 after tumor implantation (a) or at days 10, 11, and 12 (b), in two separate experiments (Exp. 1 and Exp. 2). Tumor volumes in Exp. 1 (a) and Exp. 2 (b) and animal body weight in Exp. 1 (c) 1-4 days after treatment are shown. The arrowheads in $\mathbf{c}$ indicate the time of treatment.

hypothesized that the lower efficacy of $10 \mathrm{ng}$ of NGRmTNF was related to induction of sTNF-R1 and/or sTNF-R2 and, consequently, to neutralization of its interaction with membrane receptors.

To test this hypothesis, we measured the levels of sTNF-R1 and sTNF-R2 in the serum of tumor-bearing mice, collected 1 hour after administration of various doses of mTNF and NGR-mTNF. As expected, both products induced sTNF-R2 shedding, but not sTNF-R1 shedding, at doses greater than $4 \mathrm{ng}$ (Figure 2a).

To assess whether sTNF-R2 shedding regulates the activity of NGR-mTNF, we coadministered this cytokine with $\mathrm{mAb} 6 \mathrm{G} 1$, an antagonist anti-sTNF-R2 antibody that prevents the binding of mTNF to soluble and membrane murine TNF-R2 (16). The antitumor activity of $10 \mathrm{ng}$ of NGR-mTNF was potentiated by $\mathrm{mAb} 6 \mathrm{G} 1$ (Figure $2 \mathrm{~b}$ ), in line with the hypothesis that sTNF-R2 plays a role in inhibiting the antitumor effects of NGR-mTNF.

To further support this hypothesis we compared the in vivo dose-response curve of NGR-mTNF with that of NGR-hTNF, taking advantage of the fact that the human cytokine cannot bind murine sTNF-R2 (22). We found that the dose-response curve of NGR-hTNF was not bell-shaped and that $10 \mathrm{ng}$ of NGR-hTNF is as active as $1 \mathrm{ng}$ (Figure 2c). It is also remarkable that $1 \mathrm{ng}$ was sufficient to induce the maximum antitumor effect. This may suggest that receptor binding on vessels can be achieved with very low blood levels of NGR-hTNF.

a
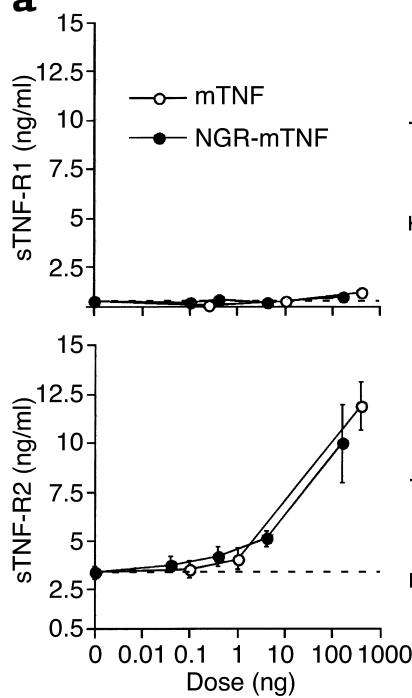

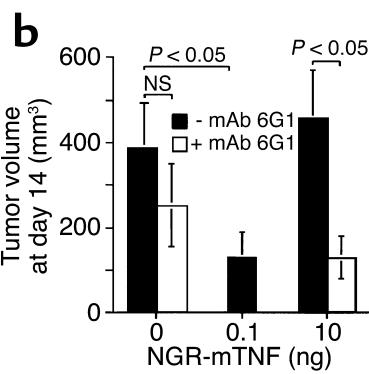

C

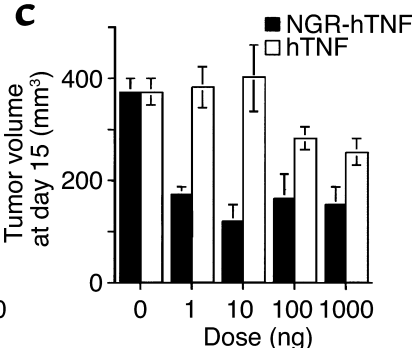



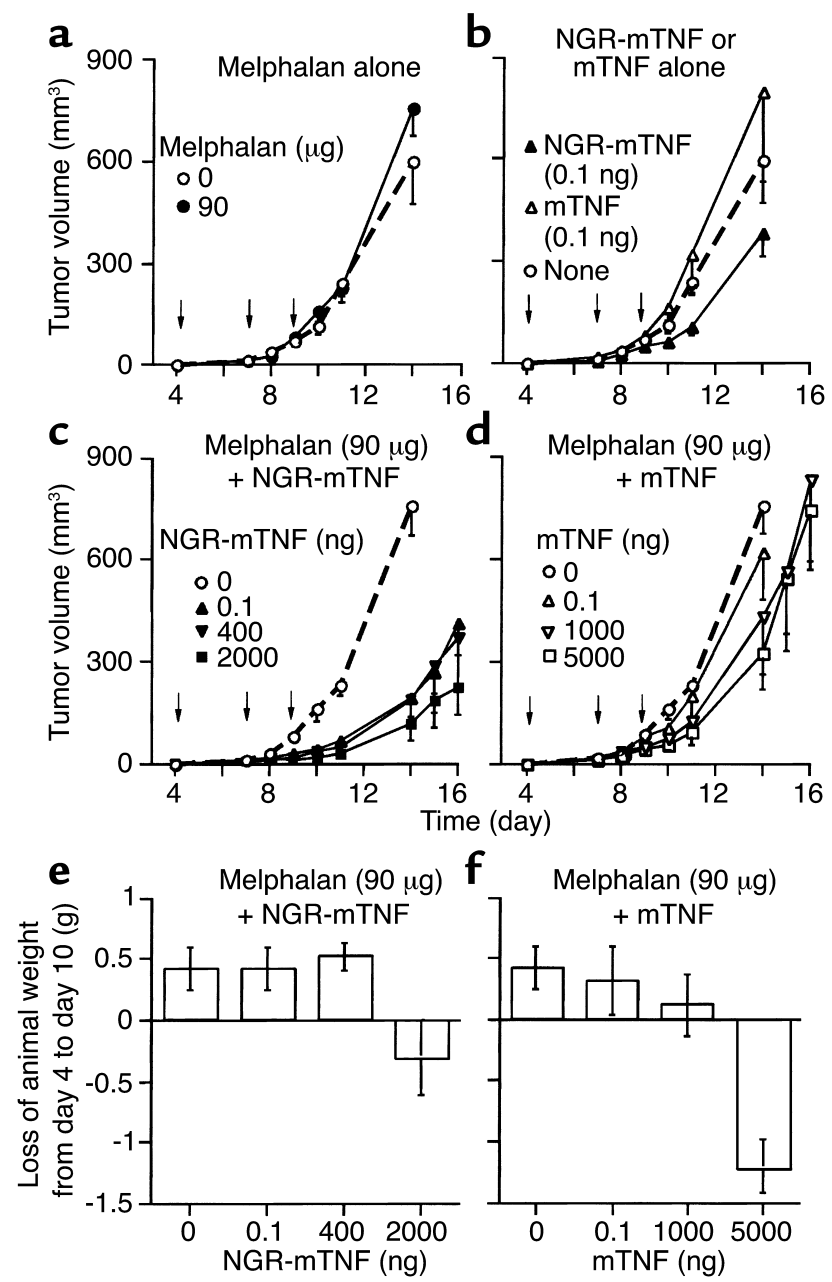

Figure 3

Effect of melphalan, alone (a) or in combination with NGR-mTNF (c) or mTNF (d), on the tumor growth (a-d) and body weight (e and f) of mice bearing B16F1 melanoma. The animals were treated intraperitoneally with the drugs and the doses indicated in each panel (five animals per group) at days 4, 7, and 9 after tumor implantation (indicated by arrows).

Taken together, the results of these experiments strongly suggest that NGR-mTNF and mTNF, at doses greater than $4 \mathrm{ng}$, induce shedding of sTNF-R2 in amounts sufficient to inhibit their antitumor activity. Picogram doses of NGR-mTNF are sufficient to enhance the therapeutic effect of melphalan and doxorubicin. We then investigated whether targeted delivery of low doses of NGR-mTNF to tumor vessels could enhance the antitumor activity of chemotherapeutic drugs. These experiments were conducted using the B16F1 model, a spontaneous mouse melanoma characterized by scarce immunogenicity and low sensitivity to melphalan, and using the RMA-T model. Melphalan $(90 \mu \mathrm{g})$ was unable to affect the growth of B16F1 tumors when injected alone (Figure 3a). Similarly, mTNF ( $0.1 \mathrm{ng}$, alone, intraperitoneally) was virtually inactive, while the same dose of NGR-mTNF modestly delayed the tumor growth (Figure $3 \mathrm{~b}$ ). The combination of melphalan with $0.1 \mathrm{ng}$ of NGR-mTNF induced stronger antitumor effects than did the single agents, indicating a synergistic effect (Figure 3c). Remarkably, the combination of melphalan with $0.1 \mathrm{ng}$ of NGR-mTNF was more effective than the combination with 5,000 ng of mTNF (Figure 3, $\mathrm{c}$ and d). We observed this synergism even when NGR-mTNF $(0.1 \mathrm{ng})$ was injected intravenously (not shown).

Two similar experiments were conducted with doxorubicin in the B16F1 model. Animals were treated 5 days after tumor implantation, with NGR-TNF, diluted in $0.9 \%$ sodium chloride containing $100 \mu \mathrm{g} / \mathrm{ml} \mathrm{HSA}$, or with diluent alone, and 2 hours later with various doses of doxorubicin (20-320 $\mu \mathrm{g}$ intraperitoneally). In both experiments, the effect of doxorubicin plus NGR-mTNF was stronger than that of doxorubicin alone (Figure 4, $a, b$, and e), indicating that NGR-mTNF markedly improves the efficacy of this drug. For example, the effect of doxorubicin $(40 \mu \mathrm{g})$ plus NGR-mTNF $(0.1 \mathrm{ng})$ was stronger than that of $320 \mu \mathrm{g}$ of doxorubicin alone (Figure $4 \mathrm{~b})$, while the effect of doxorubicin $(20 \mu \mathrm{g})$ plus NGR-mTNF was weaker (Figure 4a). From these results we estimate that the activity of doxorubicin is potentiated eight- to tenfold by NGR-mTNF in this model.

In another series of experiments, we measured the effect of NGR-mTNF in combination with melphalan or doxorubicin, administered 10-12 days after tumor implantation, i.e., when the tumors were well established. Synergism between low doses of NGR-mTNF and chemotherapy was observed in both B16F1 and RMA-T models (Figure 5). Of note, a single treatment with NGR-TNF plus melphalan cured three out of five RMA-T tumor-bearing mice. In contrast, no cure was observed with B16F1 tumors treated with NGR-mTNF, combined with either doxorubicin or melphalan. Like$l y$, this reflects the stronger immunogenicity of the Raucher virus-induced lymphoma (RMA-T) compared with the spontaneous B16F1 melanoma.

In conclusion, these results suggest that picogram doses of NGR-TNF are sufficient to improve the response of tumors to melphalan and doxorubicin.

Low doses of NGR-mTNF are not toxic and do not increase the toxicity of melphalan. To estimate the efficacy/toxicity ratio of each treatment, we monitored animal body weight daily and animal survival after treatment. While therapeutic doses of mTNF $(10,000 \mathrm{ng})$ induced marked loss of body weight in RMA-T-bearing animals (Figure 1c, left), therapeutic doses of NGR-mTNF (0.01-1 ng) did not cause loss of body weight nor animal death (Figure 1c, right). The toxicity of NGRmTNF in combination with melphalan was then examined. Three out of ten mice bearing the RMA-T tumor died 3 days after treatment with $200 \mu \mathrm{g}$ of melphalan alone. Neither NGR-mTNF nor mTNF (1 ng each) increased the lethality of melphalan $(200 \mu \mathrm{g})$, as in both cases only two out of ten animals died.

In the B16F1 model, therapeutic doses of NGRmTNF $(0.1 \mathrm{ng})$ did not cause loss of body weight, even when combined with melphalan (Figure $3 e$ ). In contrast, melphalan combined with therapeutic doses of 
a

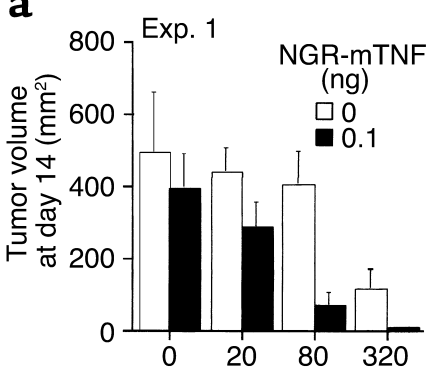

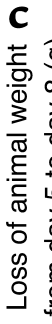

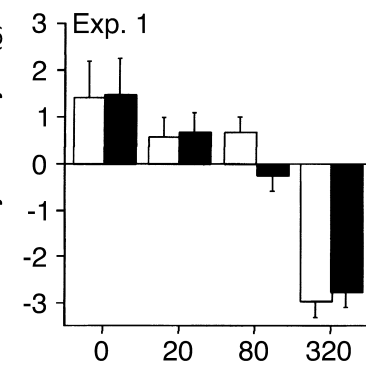

b

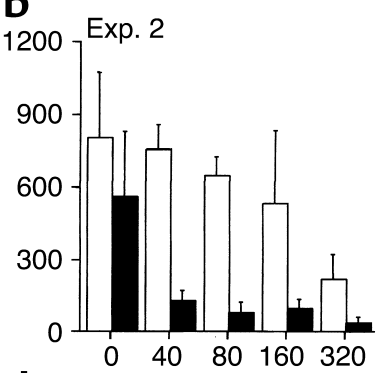

d

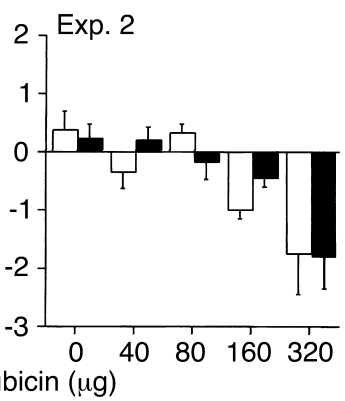

e

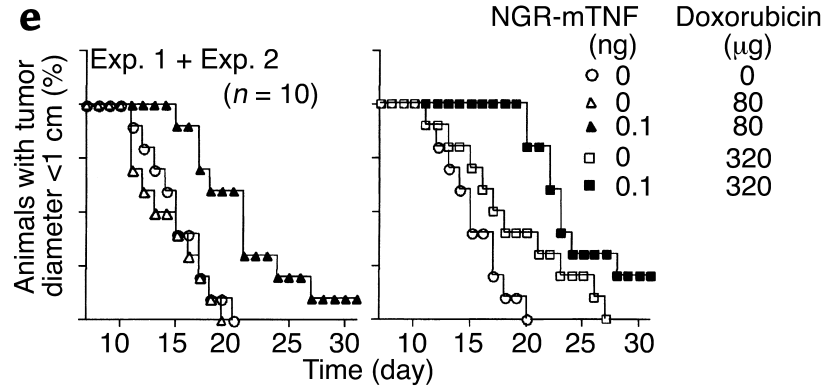

mTNF $(5 \mu \mathrm{g})$ induced marked loss of body weight (Figure $3 \mathrm{f})$. In addition, NGR-mTNF $(0.1 \mathrm{ng})$ did not increase the loss of body weight caused by high doses of doxorubicin (Figure 4, $\mathrm{c}$ and d).

These results suggest that picogram doses of NGRmTNF increase the response of tumors to melphalan and doxorubicin with no evidence of increased toxicity.

TNF-R1 activation is necessary and sufficient for the synergism between NGR-TNF and chemotherapeutic drugs. The mechanisms of the synergism between low doses of NGR-mTNF and chemotherapy were then investigated.

To assess whether these mechanisms rely on TNF-Rs activation, we tested the effect of $\mathrm{mAb} V 1 \mathrm{q}$, a neutralizing anti-mTNF antibody, on the antitumor activity of NGR-mTNF $(0.1 \mathrm{ng})$ in combination with melphalan (90 $\mu \mathrm{g}) . \mathrm{V1q}$ inhibited, at least partially, the antitumor activity of these drugs in the B16F1 model (Figure 6a). This suggests that the interaction between the TNF moiety and TNF-Rs is critical for the activity of the conjugate.

\section{Figure 5}

Effect of melphalan or doxorubicin, alone or in combination with NGR-mTNF, on well-established RMA-T and B16F1 tumors. Each animal was treated with the drugs and the doses indicated in each panel (five animals per group) at time points indicated by the arrows. The numbers on each curve indicate the animals that were tumorfree at day 43.

\section{Figure 4}

Effect of various doses of doxorubicin, alone (white bars) or in combination with NGR-mTNF (black bars), on the tumor growth (a and b), body weight (c and d), and survival (e) of mice bearing B16F1 melanomas. The drugs were administered to the animals (five mice per group intraperitoneally) 5 days after tumor implantation.

The role of TNF-R1 and TNF-R2 was then studied. To this end, we evaluated the effect of melphalan in combination with $0.01 \mathrm{ng}$ or $0.1 \mathrm{ng}$ of NGR-hTNF, a TNF-R1-specific agonist (22). The effect of melphalan in the B16F1 model was potentiated by NGR-hTNF (Figure 6b), suggesting that TNF-R1 activation is sufficient for the synergism.

The synergy between NGR-mTNF and chemotherapy is not dependent on tumor cell cytotoxicity. To assess whether the synergism depends directly on cytotoxicity against tumor cells, we measured the effect of each compound, alone or in combination, on cultured B16F1 cells. Neither melphalan nor NGR-mTNF, alone or in combination, killed these cells in a 48-hour in vitro assay (not shown). Similarly, NGR-mTNF did not enhance the cytotoxic activity of doxorubicin in vitro (not shown). These results suggest that the synergism observed in vivo is not directly dependent on cytotoxic effects against tumor cells and point to an indirect role of a component of the tumor stroma, e.g., the endothelial lining of tumor vessels.

$N G R-m T N F$ increases the penetration of doxorubicin in murine melanomas and lymphomas. We then investigated whether NGR-mTNF could increase the penetration of chemotherapeutic drugs in tumors. To this aim we measured the amount of doxorubicin that had penetrated B16F1 and RMA-T tumors 2 hours after administration, taking advantage of the fluorescent properties of this drug (23). Preliminary

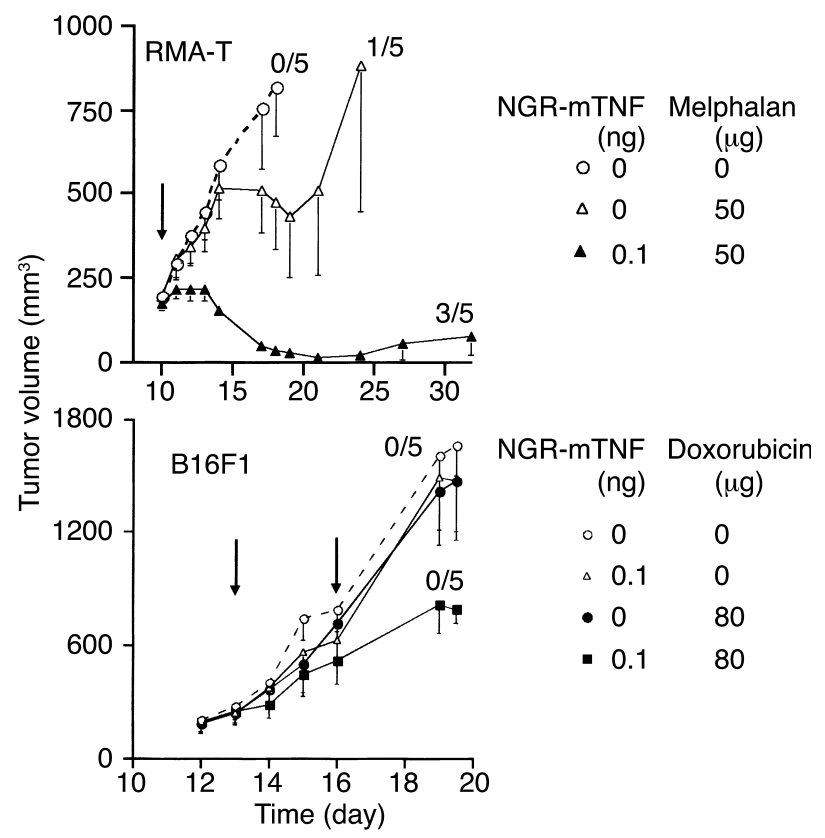




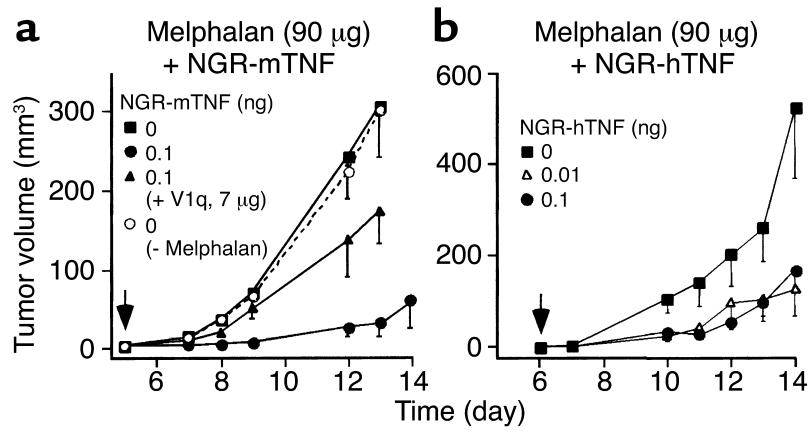

experiments showed that the nuclei of B16F1 cells become fluorescent after these cells are exposed to doxorubicin in vitro (Figure 7a). The fluorescence signal is dose-dependent and stable for at least 24 hours, when the cells are fixed with formaldehyde and kept at $4^{\circ} \mathrm{C}$, as measured by FACS (Figure $7 \mathrm{~b}$ ). Thus, the fluorescence intensity of tumor cells recovered from animals after treatment is an indication of the amount of doxorubicin that has penetrated tumors. We observed that $0.1 \mathrm{ng}$ of NGR-mTNF, administered 2 hours before doxorubicin, increased the fluorescence intensity and the percentage of positive cells recovered from both B16F1 and RMA-T tumors 2 hours after treatment (two- to fivefold;

\section{Figure 6}

Role of TNF receptors in the synergistic activity of NGR-mTNF and melphalan. (a) Effect of mAb V1q (an anti-mTNF neutralizing antibody) on the antitumor activity of melphalan in combination with NGR-mTNF in the B16F1 model. The drugs were administered at day 5. V1 $\mathrm{q}$ and NGR-mTNF were premixed and incubated for 1 hour before injection into animals. (b) Effect of melphalan in combination with NGR-hTNF at the indicated doses.

Figure $7, \mathrm{c}-\mathrm{h})$. This suggests that NGR-mTNF increased the number of cells that were reached by doxorubicin as well as the intracellular amount of drug.

\section{Discussion}

Alteration of vascular permeability and interstitial pressure, endothelial cell damage, and fibrin deposition are important mechanisms for the antitumor activity of TNF, either alone or in combination with chemotherapeutic drugs. After infusion in animals or patients, TNF can also induce negative feedback mechanisms that neutralize most of these effects. For example, TNF, even at moderate doses, can induce the release of soluble p55 and p75 TNF receptors that may prevent its interaction with membrane receptors $(21,24)$. Although these soluble inhibitors may protect the body from the harmful effects of this cytokine, they may also prevent its antitumor activity and could explain, in a

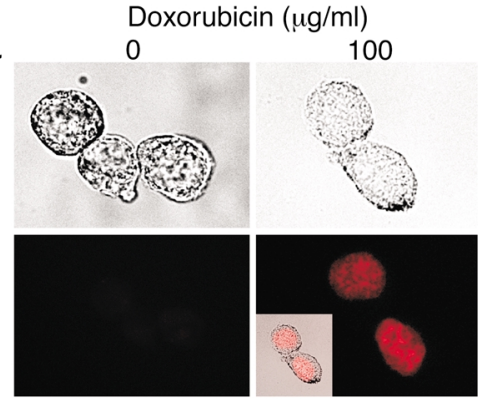

b

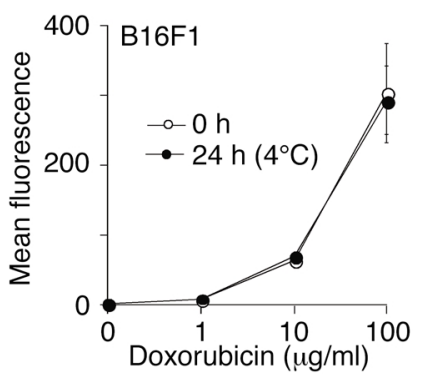

C

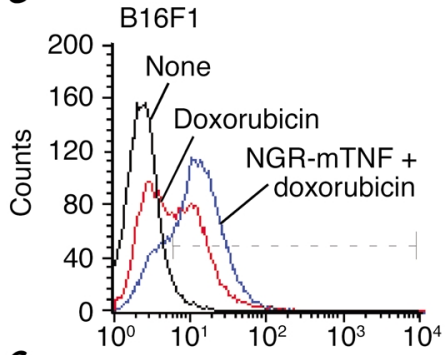

f

RMA-T

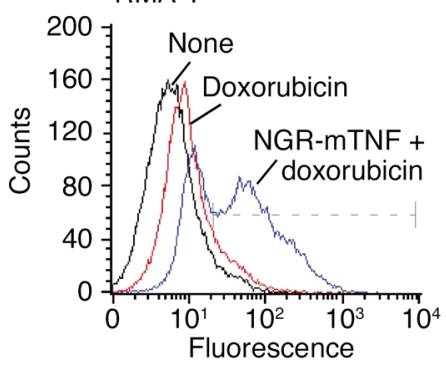

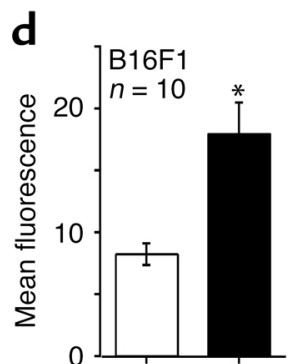
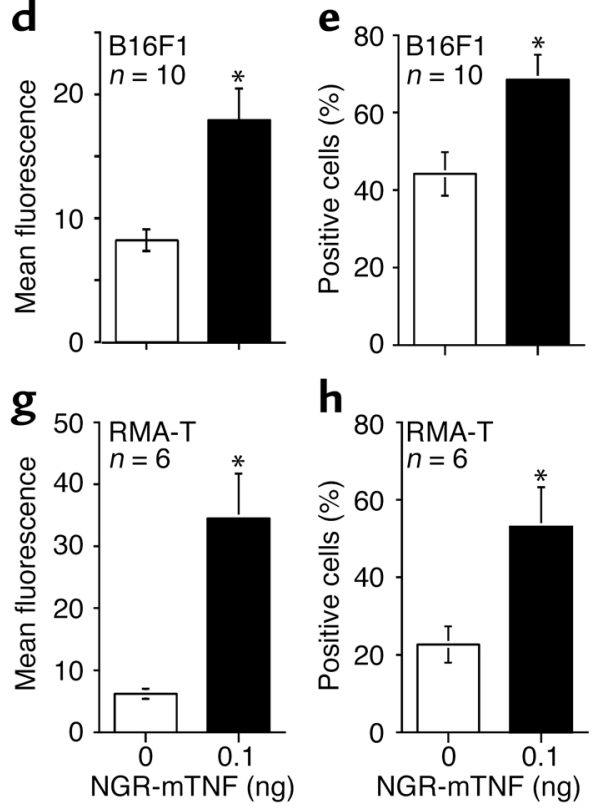

$\mathrm{h}$

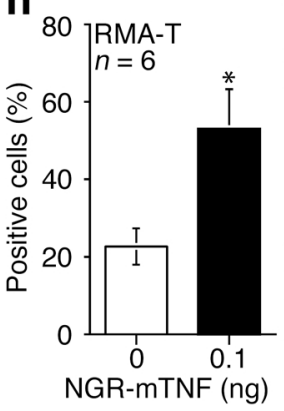

Figure 7

Effect of NGR-mTNF on the penetration of doxorubicin in B16F1 and RMA-T tumors. (a) Bright-field (upper panels) and fluorescence (lower panels) microscopy of B16F1 cells incubated in vitro with $100 \mu \mathrm{g} / \mathrm{ml}$ doxorubicin $\left(30\right.$ minutes, $\left.37^{\circ} \mathrm{C}\right)$. Inset: Merge of bright-field and fluorescence images. (b) Stability of the B16F1 fluorescence signal after in vitro treatment with doxorubicin. B16F1 cells were incubated with various doses of doxorubicin in culture medium $\left(30\right.$ minutes, $37^{\circ} \mathrm{C}$ ), washed with $0.9 \%$ sodium chloride, and fixed with $4 \%$ formaldehyde. The cells were then incubated for 0 hours or 24 hours in culture medium at $4^{\circ} \mathrm{C}$, washed again, and analyzed by FACS. (c and $\mathbf{f}$ ) Representative FACS analysis of cells recovered from B16F1 (c) or RMA-T (f) tumors 2 hours after in vivo administration of doxorubicin alone (320 $\mu \mathrm{g})$ or in combination with NGR-mTNF $(0.1 \mathrm{ng})$. Dashed lines indicate the fluorescence interval considered positive. (d and $\mathbf{g}) \mathrm{Mean} \pm \mathrm{SE}$ fluorescence of B16F1 (d) or RMA-T ( $(\mathbf{g})$ cells recovered from tumors. (e and $\mathbf{h})$ Mean \pm SE of positive cells recovered from B16F1 (e) or RMA-T $(\mathbf{h})$ tumors. ${ }^{*} P<0.05$, statistical analysis by two-tailed $t$ test. 


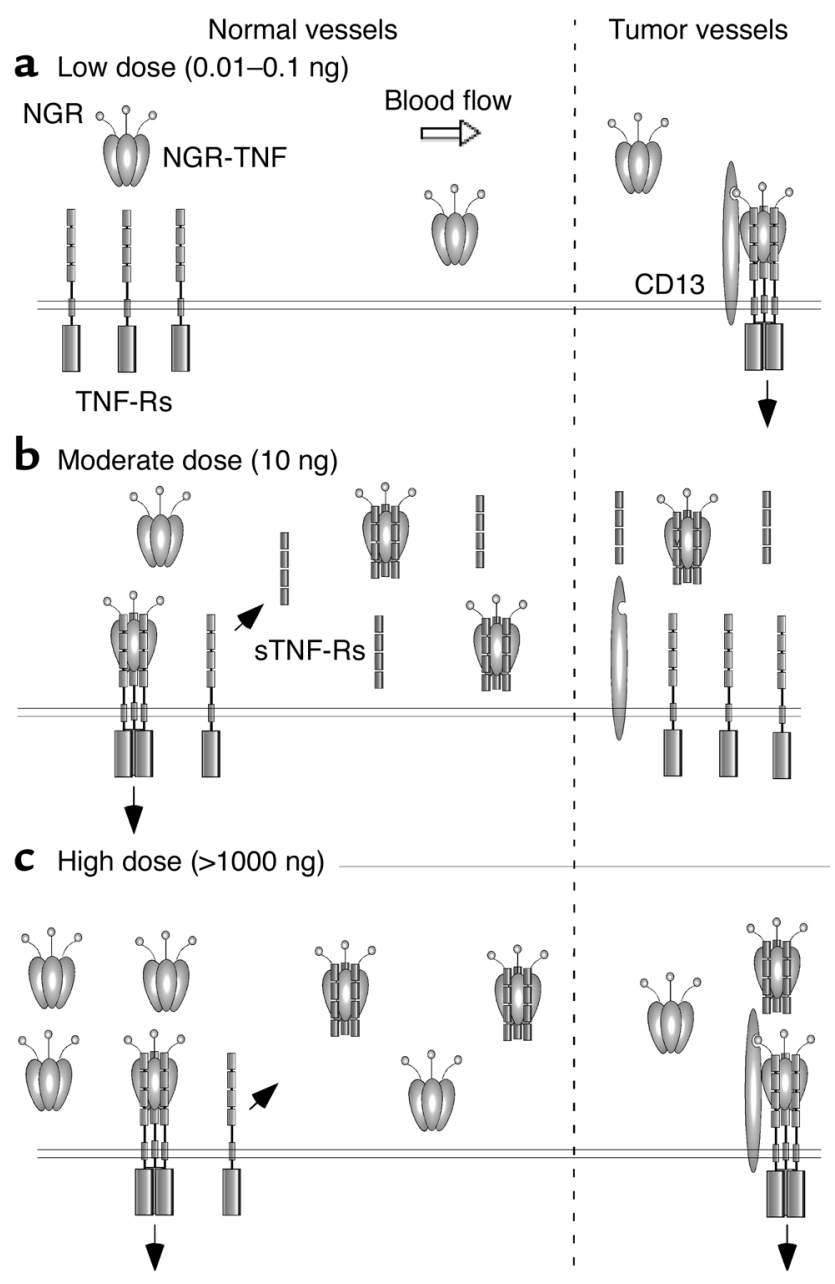

Figure 8

Schematic representation of the hypothetical interactions of low (a), moderate (b), and high (c) doses of NGR-TNF with soluble and membrane receptors in normal vessels (CD13-negative) and in tumor-associated vessels (CD13-positive). Black arrows indicate TNF receptor signaling or extracellular domain shedding.

part, the need of high doses of TNF for effective therapy. In this work we postulated that homing low doses of TNF to tumor vessels represents a new strategy to avoid toxic reactions as well as negative feedback mechanisms, while preserving its synergism with chemotherapy. To verify this hypothesis, we have investigated the antitumor activity of high and low doses of NGR-mTNF and mTNF, ranging from picogram to microgram quantities, in two murine models based on subcutaneous RMA-T lymphoma and B16F1 melanoma tumors. The study was carried out using these cytokines alone or in combination with melphalan or doxorubicin. While mTNF was virtually inactive in these models at doses lower than 100-1,000 ng, we found that NGR-mTNF, even alone, could induce antitumor effects with doses as low as $0.01-0.1 \mathrm{ng}$. Since the $\mathrm{LD}_{50}$ values of $\mathrm{mTNF}$ and NGR-mTNF are similar and correspond to about $50,000 \mathrm{ng}(2.5 \mathrm{mg} / \mathrm{kg})$ in RMA-T tumor-bearing mice (14), these results indicate that the efficacy/toxicity ratio of NGR-mTNF is $10^{4}-10^{5}$ times greater than that of mTNF.

Administration of minute amounts of NGR-mTNF (0.01-0.1 ng/mouse, $0.5-5 \mathrm{ng} / \mathrm{kg}$, about $10^{6}$-fold lower than the $\mathrm{LD}_{50}$ ) to tumor-bearing animals potentiated the antitumor activity of melphalan and doxorubicin with no evidence of increased toxicity, as judged by tumor mass reduction, animal survival, and weight loss after treatment. This suggests that NGR-mTNF improves the therapeutic index of these drugs. It is noteworthy that $5 \times 10^{4}$-fold greater doses of mTNF (e.g., $5000 \mathrm{ng} /$ mouse in the B16F1 model) were necessary to enhance the effect of melphalan to comparable levels, causing marked loss of body weight.

The fact that both melphalan and doxorubicin at doses virtually inactive in the $\mathrm{B} 16 \mathrm{~F} 1$ model reduced tumor growth when combined with NGR-mTNF indicates that these drugs act synergistically. Studies on the mechanism of action showed that the synergism relies on the interaction of NGR-mTNF with TNF-R1 on stromal cells, most likely endothelial cells, and much less on tumor cells. In addition, we found that vascular targeting with NGR-mTNF improves cytotoxic drug penetration in tumors. It is noteworthy that NGR-mTNF increased both the percentage of cancer cells that can be reached by doxorubicin in 2 hours and the intracellular amount of drug, suggesting that NGR-TNF can alter drug-penetration barriers. Previous studies showed that TNF can rapidly increase endothelial permeability $(25,26)$ and can decrease interstitial fluid pressure (8), both believed to be critical for drug penetration in tumors (1). Possibly, these mechanisms increase convective transport of drugs through tumor vessel wall and interstitium, finally resulting in increased drug uptake by tumor cells. The timing of administration is likely critical for these mechanisms, as TNF can also induce intravascular coagulation (27), leading to vessel occlusion and reduction of tumor perfusion. In keeping with this view, we observed that the effect of melphalan was higher when this drug was administered 2 hours after NGR-TNF than when it was administered after 6 hours (data not shown). Besides these mechanisms, other known effects of TNF on endothelial cells could contribute to its overall antitumor activity, including the induction of endothelial leukocyte adhesion molecules, inflammatory cytokines, chemokines, class II molecules, and procoagulant factors $(11,12)$. These mechanisms, together with improved chemotherapeutic drug penetration, could contribute to activating inflammatory and immune responses.

The hypothesis that vascular targeting could avoid negative feedback mechanisms, usually associated with TNF therapy, is supported by the observation that picogram doses of NGR-mTNF do not induce soluble receptor shedding, while both NGR-mTNF and mTNF rapidly induce the release of STNF-R2 into the circulation at doses greater than 4-10 ng. These levels of 
sTNF-R2 inhibited most of the antitumor activity of 10 ng of NGR-mTNF and may explain the paradoxical observation that $10 \mathrm{ng}$ is less active than $0.1 \mathrm{ng}$. Likely, a large proportion of injected molecules were rapidly complexed by sTNF-Rs and their activity was blocked.

The molecular mechanisms underlying the selective interaction of low doses of NGR-mTNF with tumor blood vessels have been partially elucidated. We have shown recently that different CD13 isoforms are expressed in tumor-associated vessels, in epithelia, and in myeloid cells, and that the NGR domain of NGRTNF selectively recognizes a CD13 isoform associated with tumor vessels (28). We hypothesize, therefore, that low blood levels of NGR-mTNF can rapidly interact with CD13-positive endothelial cells, because of highavidity multivalent binding involving both CD13 and TNF-Rs, and that they interact little or not at all with CD13-negative endothelial cells of normal vessels, because of lower avidity. A schematic representation of these concepts and of the hypothetical interactions of NGR-TNF with soluble and membrane receptors is shown in Figure 8.

In conclusion, we have found that targeted delivery of picogram doses of NGR-mTNF to tumor vessels enhances the antitumor activity of chemotherapeutic drugs in mice without inducing soluble TNF-Rs shedding. Given that the CNGRC motif is expected to target human as well as murine tumor-associated vessels (29), our results suggest that the combination of low doses of NGR-TNF with melphalan or doxorubicin could increase their therapeutic index in patients.

\section{Acknowledgments}

We thank Matteo Bellone for helpful discussions. This work was supported by Associazione Italiana Ricerca sul Cancro.

1. Jain, R.K. 1994. Barriers to drug delivery in solid tumors. Sci. Am. 271:58-65.

2. Lienard, D., Ewalenko, P., Delmotte, J.J., Renard, N., and Lejeune, F. 1992. High-dose recombinant tumor necrosis factor alpha in combination with interferon gamma and melphalan in isolation perfusion of the limbs for melanoma and sarcoma. J. Clin. Oncol. 10:52-60.

3. Eggermont, A.M., et al. 1996. Isolated limb perfusion with high-dose tumor necrosis factor-alpha in combination with interferon-gamma and melphalan for nonresectable extremity soft tissue sarcomas: a multicenter trial. J. Clin. Oncol. 14:2653-2665.

4. Lejeune, F.J. 1995. High dose recombinant tumour necrosis factor (rTNF alpha) administered by isolation perfusion for advanced tumours of the limbs: a model for biochemotherapy of cancer. Eur. J. Cancer. 31A:1009-1016

5. Fraker, D.L., Alexander, H.R., Andrich, M., and Rosenberg, S.A. 1996 Treatment of patients with melanoma of the extremity using hyperthermic isolated limb perfusion with melphalan, tumor necrosis factor, and interferon gamma: results of a tumor necrosis factor dose-escalation study. J. Clin. Oncol. 14:479-489.

6. Rossi, C.R., et al. 1999. Soft tissue limb sarcomas: Italian clinical trials with hyperthermic antiblastic perfusion. Cancer. 86:1742-1749.

7. van der Veen, A.H., et al. 2000. TNF-alpha augments intratumoural concentrations of doxorubicin in TNF-alpha-based isolated limb perfusion in rat sarcoma models and enhances anti-tumour effects. Br. J. Cancer. 82:973-980.

8. Kristensen, C.A., Nozue, M., Boucher, Y., and Jain, R.K. 1996. Reduction of interstitial fluid pressure after TNF-alpha treatment of three human melanoma xenografts. Br. J. Cancer. 74:533-536.

9. Suzuki, S., Ohta, S., Takashio, K., Nitanai, H., and Hashimoto, Y. 1990. Augmentation for intratumoral accumulation and anti-tumor activity of liposome-encapsulated adriamycin by tumor necrosis factor-alpha in mice. Int. J. Cancer. 46:1095-1100.

10. de Wilt, J.H., et al. 2000. Tumour necrosis factor alpha increases melphalan concentration in tumour tissue after isolated limb perfusion. $\mathrm{Br}$. J. Cancer. 82:1000-1003.

11. Fiers, W. 1995. Biologic therapy with TNF: preclinical studies. In Biologic therapy of cancer: principles and practice. V. De Vita, S. Hellman, and S. Rosenberg, editors. J.B. Lippincott Co. Philadelphia, Pennsylvania, USA 295-327.

12. Fraker, D.L., Alexander, H.R., and Pass, H.I. 1995. Biologic therapy with TNF: systemic administration and isolation-perfusion. In Biologic therapy of cancer: principles and practice. V. De Vita, S. Hellman, and S. Rosenberg, editors. J.B. Lippincott Co. Philadelphia, Pennsylvania, USA. 329-345.

13. Corti, A., and Marcucci, F. 1998. Tumour necrosis factor: strategies for improving the therapeutic index. J. Drug Target. 5:403-413.

14. Curnis, F., et al. 2000. Enhancement of tumor necrosis factor alpha antitumor immunotherapeutic properties by targeted delivery to aminopeptidase N (CD13). Nat. Biotechnol. 18:1185-1190.

15. Moro, M., et al. 1997. Tumor cell targeting with antibody-avidin complexes and biotinylated tumor necrosis factor alpha. Cancer Res. 57:1922-1928.

16. Corti, A., et al. 1999. Upregulation of $\mathrm{p} 75$ tumor necrosis factor alpha receptor in Mycobacterium avium-infected mice: evidence for a functional role. Infect. Immun. 67:5762-5767.

17. Pelagi, M., et al. 2000. Caspase inhibition reveals functional cooperation between p55- and p75-TNF receptors in cell necrosis. Eur. Cytokine Netw. 11:580-588.

18. Corti, A., Poiesi, C., Merli, S., and Cassani, G. 1994. Tumor necrosis factor (TNF) alpha quantification by ELISA and bioassay: effects of TNF alpha-soluble TNF receptor ( $\mathrm{p} 55$ ) complex dissociation during assay incubations. J. Immunol. Methods. 177:191-198.

19. Smith, R.A., and Baglioni, C. 1987. The active form of tumor necrosis factor is a trimer. J. Biol. Chem. 262:6951-6954.

20. Gasparri, A., et al. 1999. Tumor pretargeting with avidin improves the therapeutic index of biotinylated tumor necrosis factor alpha in mouse models. Cancer Res. 59:2917-2923.

21. Aderka, D., et al. 1998. Shedding kinetics of soluble tumor necrosis factor (TNF) receptors after systemic TNF leaking during isolated limb perfusion. Relevance to the pathophysiology of septic shock. J. Clin. Invest. 101:650-659.

22. Tartaglia, L.A., et al. 1991. The two different receptors for tumor necrosis factor mediate distinct cellular responses. Proc. Natl. Acad. Sci. USA. 88:9292-9296.

23. Luk, C.K., and Tannock, I.F. 1989. Flow cytometric analysis of doxorubicin accumulation in cells from human and rodent cell lines. J. Natl. Cancer Inst. 81:55-59.

24. Sella, A., et al. 1995. Phase I study of tumor necrosis factor plus actinomycin D in patients with androgen-independent prostate cancer. Cancer Biother. 10:225-235.

25. Brett, J., et al. 1989. Tumor necrosis factor/cachectin increases permeability of endothelial cell monolayers by a mechanism involving regulatory G proteins. J. Exp. Med. 169:1977-1991.

26. Goldblum, S.E., and Sun, W.L. 1990. Tumor necrosis factor-alpha augments pulmonary arterial transendothelial albumin flux in vitro. Am.J. Physiol. 258:L57-L67.

27. Clauss, M., Ryan, J., and Stern, D. 1992. Modulation of endothelial cell hemostatic properties by TNF: insights into the role of endothelium in the host response to inflammatory stimuli. In Tumor necrosis factors: the molecules and their emerging roles in medicine. B. Beutler, editor. Raven Press. New York, New York, USA. 49-63.

28. Curnis, F., et al. 2002. Differential binding of drugs containing the NGR motif to CD13 isoforms in tumor vessels, epithelia and myeloid cells. Cancer Res. 62:867-874.

29. Arap, W., Pasqualini, R., and Ruoslahti, E. 1998. Cancer treatment by targeted drug delivery to tumor vasculature in a mouse model. Science. 279:377-380. 